\title{
BMJ Open Are there non-linear relationships between alcohol consumption and long- term health? Protocol for a systematic review of observational studies employing approaches to improve causal inference
}

\author{
Rachel Visontay (D) , ${ }^{1}$ Matthew Sunderland, ${ }^{1}$ Tim Slade, ${ }^{1}$ Jack Wilson, \\ Louise Mewton ${ }^{1,2}$
}

To cite: Visontay $\mathrm{R}$ Sunderland M, Slade T, et al. Are there non-linear relationships between alcohol consumption and long-term health? Protocol for a systematic review of observational studies employing approaches to improve causal inference. BMJ Open 2021;11:e043985. doi:10.1136/ bmjopen-2020-043985

- Prepublication history and additional material for this paper is available online. To view these files, please visit the journal online (http://dx.doi.org/10. 1136/bmjopen-2020-043985).

Received 20 August 2020 Revised 07 February 2021 Accepted 18 February 2021

Check for updates

(c) Author(s) (or their employer(s)) 2021. Re-use permitted under CC BY-NC. No commercial re-use. See rights and permissions. Published by BMJ.

${ }^{1}$ The Matilda Centre for Research in Mental Health and Substance Use, The University of Sydney, Sydney, New South Wales, Australia

${ }^{2}$ Centre for Healthy Brain Ageing University of New South Wales, Sydney, New South Wales, Australia

Correspondence to

Dr Louise Mewton;

louisem@unsw.edu.au

\begin{abstract}
Introduction There is a substantial literature finding that moderate alcohol consumption is protective against certain health conditions. However, more recent research has highlighted the possibility that these findings are methodological artefacts, caused by confounding and other biases. While modern analytical and study design approaches can mitigate confounding and thus enhance causal inference in observational studies, they are not routinely applied in research assessing the relationship between alcohol use and long-term health outcomes. The purpose of this systematic review is to identify observational studies that employ these analytical/design-based approaches in assessing whether relationships between alcohol consumption and health outcomes are non-linear. This review seeks to evaluate, on a per-outcome basis, what these studies find the strength and form of the relationship between alcohol consumption and health to be.
\end{abstract}

Methods and analysis Electronic databases (MEDLINE, PsycINF0, Embase and SCOPUS) were searched in May 2020. Study selection will comply with the Preferred Reporting Items for Systematic Reviews and Meta-Analyses guidelines. Articles will be screened against eligibility criteria intended to capture studies using observational data to assess the relationship between varying levels of alcohol exposure and any long-term health outcome (actual or surrogate), and that have employed at least one of the prespecified approaches to enhancing causal inference. Risk of bias of included articles will be assessed using study design-specific tools. A narrative synthesis of the results is planned.

Ethics and dissemination Formal ethics approval is not required given there will be no primary data collection. The results of the study will be disseminated through published manuscripts, conferences and seminar presentations.

PROSPERO registration number CRD42020185861.
Strengths and limitations of this study

- This systematic review will be the first to identify those observational studies that best promote causal inference when assessing the relationship between alcohol and health outcomes, promising an important contribution to the literature.

- A strength is that we will be searching for a broad, comprehensive range of analytical and designbased approaches to improving causal inference.

- A further strength is the examination of a broad range of long-term health outcomes.

- Given considerable differences among health outcomes and methodological approaches, analysis will be limited to narrative synthesis.

\section{INTRODUCTION}

Despite the significant contribution of alcohol to the burden of disease worldwide, ${ }^{1}$ there is a substantial literature finding that low-tomoderate alcohol consumption is associated with improved health for a wide range of outcomes, including cardiovascular, ${ }^{2}$ cognitive, ${ }^{3}$ diabetic ${ }^{4}$ and mental health ${ }^{5}$ conditions, as well as mortality itself. ${ }^{6}$ Here, a 'J-shaped' relationship is common, where moderate consumption represents the nadir for risk, compared with a somewhat elevated risk for those who abstain from alcohol and a greatly increased risk for heavy alcohol drinkers.

However, concerns with this literature have been raised, such as findings of protection against certain outcomes (eg, cirrhosis of the liver) that currently have no known plausible biological mechanism. ${ }^{7}$ Such criticisms have prompted scrutiny of biases in observational studies, raising the possibility that these J-shaped relationships are simply 
methodological artefacts. ${ }^{7} 8$ Methodological limitations in this literature include confounding and reverse causality-issues that plague epidemiological research more generally in efforts to identify and estimate causal relationships-as well as selection biases and measurement errors more specific to this research question. ${ }^{8-10}$

Many of these biases would be obviated by randomised controlled trials (RCT) in which participants are randomly assigned to different consumption levels and followed up on health outcomes. But due to practical and ethical considerations, no long-term RCTs have been conducted. In short-term interventional studies, moderate alcohol consumption has been found to promote beneficial changes in several biomarkers of cardiovascular health. ${ }^{11}$ Similarly, a 2-year RCT of diabetics found moderate wine consumption led to reduced cardiometabolic risk. ${ }^{12}$ So, while this provides some evidence for the benefits of moderate alcohol consumption, such benefits may only be transitory, and harms not yet apparent. There is also a dearth of RCTs investigating a reduction in alcohol consumption to abstention specifically, ${ }^{13}$ particularly in those who are moderate consumers. Those reduction trials that have been conducted tend to be short-term and have limited applicability to the research question at hand. Alcohol consumption can have complex cumulative and or/delayed effects ${ }^{4}$; without long-term follow-up, these cannot be observed.

Instead, recent efforts have focused on mitigating biases in observational designs, with a focus on the impact of methodological decisions regarding data collection and analysis. There is acknowledgement that such decisionsfor example, which covariates to measure, how exposure levels are defined and compared, which population to sample, what model to use-can have substantial effects on conclusions about the strength and form of a relationship. ${ }^{145}$ Tools available in the study planning phase can improve some of these methodological decisions. For example, causal diagrams can be created to identify confounders as well as possible selection bias. Steps implemented post-main analyses can help to determine the robustness of findings across alternative methodological decisions ('sensitivity analyses'), or in the face of potential unmeasured bias ('bias analysis'). Additional checks for robustness across populations with different underlying confounding structures ('cross-cohort comparison') or across different methodological approaches ('triangulation') can also strengthen causal conclusions. ${ }^{16}$

Modern epidemiological developments in data analysis and alternative study designs, however, may have the most potential to mitigate the limitations of existing research, particularly in addressing confounding. Causal inference in conventional observational designs (eg, prospective cohort studies) can be enhanced with modern data analysis methods. These include propensity scores (estimating the exposure mechanism as a data preprocessing step prior to main analyses) used primarily for matching or inverse probability weighting, and 'G-methods' (which can account for time-varying variables). Doubly robust methods incorporate estimation of both the exposure and outcome mechanisms, so only require correct specification of one of these models to produce accurate estimates of an exposure's effect. ${ }^{17}$ Importantly, these methods still rely on all relevant confounders being measured, and measured without error (ie, residual confounding is still possible). Other analytical tools that may enhance causal inference include fixed effects regression (focusing exclusively on within-subject variation, thus avoiding confounding) and causal mediation (identifying the mechanism/s through which an exposure may be exerting a causal effect).

Alternate observational study designs represent another approach, often eschewing the need to accurately identify and measure confounders altogether. Natural experiments, including those based on instrumental variables, mimic the random allocation of an RCT, bypassing the problem of confounding, and guarding against reverse causation. Genetic instrumental variable designs such as Mendelian randomisation (MR) show particular promise because of their ability to find proxies for alcohol consumption, and are increasingly popular in this research area. Family based designs can also mitigate unmeasured confounding (to the extent to which relevant covariates are shared), as can negative control designs. ${ }^{18}$ Table 1 provides an overview of these analytical and alternative design methods for improving causal inference.

As other sources of bias are addressed with approaches that consist largely of considered, literature-informed researcher decisions, they do not lend themselves to terminology for a systematic database search and are not the primary focus of this review. However, some of the included methods of interest do mitigate other biases in addition to confounding, for example, MR counters reverse causation and measurement error.

\section{Rationale}

The functional forms of many of the relationships between alcohol use and specific health outcomes are currently unclear. Several recent pooled analyses and reviews have failed to provide evidence for J-shaped relationships, finding monotonically increasing dose-response relationships with most health outcomes, including most, if not all, cancers. ${ }^{19}$ Yet certain cardiovascular and diabetic conditions are consistent exceptions, ${ }^{19}$ and new, rigorous individual studies that consider the impact of methodological decisions continue to find J-shaped relationships for other outcomes, for example, mortality. ${ }^{6}$ Whether these findings reflect truly causal relationships remains an open question.

The state of the evidence base may be due to the fact that, while some of the discussed analytical and design-based methods are becoming more popular in health research, ${ }^{20}$ they are not frequently employed in this specific research area (with calls for greater implementation ${ }^{21}$ ). Despite the many reviews focusing on the relationship between alcohol use and various health outcomes, analytical methods to 
Table 1 Description of methods to enhance causal inference of interest for this systematic review

\begin{tabular}{|c|c|}
\hline Method & $\begin{array}{l}\text { Relevant } \\
\text { submethods }\end{array}$ \\
\hline \multicolumn{2}{|c|}{ Analytical methods applied to traditional longitudinal study desi } \\
\hline Propensity scores (PS) ${ }^{33} 34$ & $\begin{array}{l}\text { Covariate } \\
\text { balancing } \\
\text { propensity scores }\end{array}$ \\
\hline \multirow[t]{2}{*}{ G-methods ${ }^{20} 35-37$} & \\
\hline & $\begin{array}{l}\text { G-formula } \\
\text { (aka G- } \\
\text { computation or G- } \\
\text { standardisation) }{ }^{17} \\
203538\end{array}$ \\
\hline
\end{tabular}

Description

Analytical methods applied to traditional longitudinal study designs

$\begin{array}{ll}\text { Covariate } & \text { The PS is a single value reflecting the probability of exposure } \\ \text { balancing } & \text { for an individual given their values on all relevant covariates. } \\ \text { propensity scores } & \text { PS generation occurs as a data 'preprocessing' step prior to }\end{array}$ main analysis.

- Usually generated via logistic regression.

- Once generated, the PS can be used for matching, stratification, weighting (using inverse probability of treatment weights) or as a covariate for adjustment in regression.

- A family of methods intended for use with time-dependent variables.

- Developed as a solution to the problem of time-varying covariates affected by past exposure, including those that act as both confounders and mediators over time.

- The three G-methods are the G-formula, marginal structural models and G-estimation, each relying on its own modelling assumptions.

- First models relationship given observed data (using actual exposure for each individual), and then predicts outcomes under counterfactual exposures, with the difference taken as the causal effect.

- Is a generalisation of standardisation (conditioning on covariates and then marginalising) that accounts for dynamic variables by considering covariate distribution over followup time.

Marginal structural - Use weights based on inverse probability of exposure at models $(\mathrm{MSMs})^{20} \quad$ each time point to create a pseudo-population, where 3538 each combination of covariates is equally present in each exposure condition.

- Using these weights, MSMs then estimate the causal effect.

- The most popular of the G-methods.

G-estimation of structural nested models 353738

- At each wave assesses the relationship between exposure and likelihood of outcome given covariates, adjusting for exposure and covariate values from past waves, thus accounting for dynamic confounders affected by past exposure.

- Considered semi-parametric in that mean counterfactual outcomes under no exposure are unspecified.

$\begin{array}{ll}\text { Doubly robust methods }^{17} & \text { Targeted } \\ & \text { maximum } \\ & \text { likelihood } \\ & \text { estimation } \\ \text { Augmented } & \text { inverse probability } \\ \text { weighting }\end{array}$

Fixed effects regression ${ }^{39-41}$
- Incorporates both an estimation of the outcome mechanism (as in G-formula or regression adjustment) and the exposure mechanism (as in propensity scores).

A technique developed in the econometrics literature for use with longitudinal data with repeat outcome measurements, only using information on within-subject variation, thus controlling for all time-invariant sources of confounding.

- Treats time-invariant characteristics that differ between individuals as fixed parameters (unlike in mixed models), allowing estimation of parameters of interest net of stable confounders.

- Each participant serves as own control. 
Table 1 Continued

\begin{tabular}{|c|c|c|}
\hline Method & $\begin{array}{l}\text { Relevant } \\
\text { submethods }\end{array}$ & Description \\
\hline Causal mediation analysis ${ }^{42-44}$ & & $\begin{array}{l}\text { Integrates traditional mediation analysis (which separately } \\
\text { estimates total effect of exposure on outcome, indirect effect } \\
\text { via mediators and direct effect unexplained by mediators) } \\
\text { with the potential outcomes framework to allow for } \\
\text { exposure-mediator interaction and non-linear relationships } \\
\text { (ie, is a non-parametric method). } \\
\text { Uses the concepts of 'controlled direct effect', 'natural direct } \\
\text { effect' and 'natural indirect effect'. } \\
\text { Makes explicit underlying assumptions related to } \\
\text { unmeasured confounding, and encourages sensitivity } \\
\text { analyses to test robustness to assumption violations. }\end{array}$ \\
\hline
\end{tabular}

Alternative observational study designs

Natural experiments ${ }^{1645-47}$

Standard natural experiments Instrumental variable analysis
Mimic randomised controlled trials by exploiting exogenous events that are truly randomised/approximate random assignment.

- Differ from true experiments in that exposure is not assigned by the researcher.

- Assignment may be as a result of naturally occurring phenomena (eg, a weather event), or of human intervention implemented for reasons other than the research question (eg, army draft lottery).

- Natural experiments where individuals are as-if/randomly assigned to exposure and control groups.

- Assesses the relationship between an as-if/ randomly assigned proxy for the exposure of interest and the outcome.

- A valid instrumental variable must be associated with the exposure of interest, be independent of confounders of the exposure-outcome relationship and should affect the outcome only via the exposure.

Genetic instrumental variables

- Subset of instrumental variable analysis using genetic variants as proxies for exposure.

- The most prominent technique is Mendelian Randomisation.

\begin{tabular}{|c|c|c|}
\hline Quasi-experiments ${ }^{45}$ & & $\begin{array}{l}\text { Like natural experiments, exploit exogenous events to } \\
\text { assess relationships between exposures and outcomes, but } \\
\text { lack random or as-if random assignment. }\end{array}$ \\
\hline Family based designs ${ }^{1646}$ & $\begin{array}{l}\text { Twin studies } \\
\text { Sibling } \\
\text { comparison }\end{array}$ & $\begin{array}{l}\text { By comparing genetically related participants discordant } \\
\text { for the exposure of interest, accounts for confounding from } \\
\text { genetic or shared environmental sources. }\end{array}$ \\
\hline Negative controls ${ }^{1846}$ & $\begin{array}{l}\text { Negative control } \\
\text { exposures } \\
\text { Negative control } \\
\text { outcomes }\end{array}$ & $\begin{array}{l}\text { Have the same confounding structures as the exposure- } \\
\text { outcome relationship of interest, but lack a plausible causal } \\
\text { mechanism. } \\
\text { If association is greater for the relationship of interest than } \\
\text { for the negative control, a causal relationship is likely; if not, } \\
\text { suggests confounding/other shared biases responsible. } \\
\text { May take the form of a negative control exposure or a } \\
\text { negative control outcome. }\end{array}$ \\
\hline
\end{tabular}

counter confounding are rarely a focus. Similarly, reviews of observational research tend to search exclusively for studies with traditional designs (eg, cohort studies), meaning findings from novel study designs are not integrated. As such, the planned review, focusing on those studies that best promote causal inference by employing methods that mitigate confounding, comprises an original and important contribution.

\section{Objective}

The objective of this review is therefore to identify all observational studies that have employed the aforementioned 
analytical or design-based approaches (see table 1), and to synthesise their findings on the strength and functional form (ie, support for non-linearity) of the relationships between levels of alcohol consumption and various longterm health outcomes (or surrogates) in humans.

\section{METHODS}

This protocol complies with the Preferred Reporting Items for Systematic Reviews and Meta-Analysis Protocols (PRISMA-P) statement, ${ }^{22}$ the checklist for which can be found in online supplemental table 1 . This protocol has been registered with the PROSPERO International Prospective Register of Systematic Reviews of the University of York (CRD42020185861). Reporting of the systematic review will be informed by PRISMA (online supplemental material $1^{23}$ ).

\section{Search strategy}

Electronic databases (MEDLINE, PsycINFO, Embase and Scopus) were searched in May 2020 for peer-reviewed, English-language journal articles, with no restrictions on date range. Relevant grey literature returned by Scopus will also be considered. The search strategy was designed to capture all studies using observational data to assess the relationship between varying levels of alcohol exposure and any health outcome, and that have employed at least one of the prespecified approaches to improving causal inference.

Searches ran a combination of MeSH or other controlled vocabulary terms as well as free-text words. Five groups of terms are used in the search strategy, relating to: (1) alcohol; (2) levels or patterns of drinking; (3) observational, longitudinal studies; (4) approaches to improve causal inference that are used in conjunction with observational, longitudinal study designs and (5) approaches to improve causal inference that may be considered their own study designs. These concepts are combined with the following logic: 1 and 2 and ( ( 3 and 4$)$ or 5$)$. Search terms were adapted from recent systematic reviews and from keywords/indexing of a set of key eligible papers known to the study authors. These terms were iteratively refined to improve sensitivity by cross-checking results against the set of key papers. The MEDLINE search terms are provided in online supplemental table 2.

Cross-checking of reference lists of relevant, retrieved publications will be used to supplement the database searches.

\section{Eligibility criteria}

Eligibility criteria for this review are spread across population, exposure, comparator, outcome and study design components of the publications under consideration (PI/ECOS).

\section{Population}

Only human studies will be eligible for inclusion. While there are no other restrictions on eligible populations, alcohol consumption must be assessed in the same individuals to which the health outcome/s accrue (ie, the relationship between an individual's alcohol consumption and the health of others is beyond the scope of this review).

\section{Exposure}

The exposure of interest is level of alcohol consumption (volume over a certain period) or level and pattern of consumption (incorporating information on frequency or presence of heavy episodic drinking). Studies must have discretised level of alcohol consumption into categories. This is typical practice for this research question, as analyses involving comparisons between such groups do not make assumptions about functional form (unlike analyses of continuous predictors). Specifically, there must be a non-drinking reference group and at least two other levels of consumption (or alternative structure in instrumental variables designs allowing for tests of non-linear relationships). While it is anticipated that the construction of these categories will vary (eg, different criteria for volume/frequency of consumption, differential use of lifetime and current consumption), all will be eligible for inclusion. Differences between studies in drinking category composition, such as whether former drinkers are categorised separately from lifetime abstainers, will be considered during data synthesis.

\section{Comparator}

Reference groups should comprise abstainers/nondrinkers, but reference groups partly or solely comprised occasional drinkers will also be accepted. Non-drinker reference groups that include former drinkers will also be accepted. Differences between studies in reference group composition will be considered during data synthesis.

\section{Outcomes}

Studies on the association between alcohol consumption and health outcomes have reported J-shaped relationships for a wide range of long-term conditions and their surrogates (eg, biomarkers). The focus of this review is to examine how approaches to improving causal inference in observational research have been used across the entirety of the literature, regardless of outcome type. Given common targets of study in the broader literature, it is anticipated that papers included in this review will include the following outcomes: cancer (and subtypes), cardiovascular disease (and subtypes), cognition and dementia, mental health conditions, diabetes and causespecific or all-cause mortality, among others. Short-term or acute conditions such as injury, acute exacerbation of mental illness, sexually transmitted diseases, alcohol-drug interactions and sexual function will be excluded.

\section{Study design}

Observational studies assessing the relationship between varying levels of alcohol consumption and any health outcome will be eligible. Comparisons between consumption categories must have allowed for the detection of 
non-linear relationships and must have employed at least one of the prespecified analytical/design-based approaches. Incorporating feedback from academics with expertise in causal inference methods, the approaches of explicit interest chosen for this review have been clearly defined a priori and have been recognised and employed in recent years for their potential to enhance causal inference. Specifically, these are: propensity scores, the G family of methods, targeted maximum likelihood estimation and other doubly robust methods, fixed effects regression, causal mediation analysis, standard natural experiments, MR and other instrumental variables, quasiexperiments, family-based methods and negative controls.

Conventional observational studies employing analytical methods to promote causal inference must be cohort or case-control designs and must be longitudinal, with alcohol consumption measured at least once at a time point preceding measurement of health outcome/s (studies employing MR/other natural experiment approaches will be eligible even if not longitudinal). Systematic reviews/meta-analyses, interventional studies and animal studies will be excluded.

\section{Study selection}

All citations will be imported into Endnote ${ }^{24}$ and deduplicated. Citations will then be uploaded to Covidence, ${ }^{25}$ which will be used to facilitate screening. Titles and abstracts returned by the database searches (and any additional papers identified by hand) will be screened independently by one reviewer (RV), with a second reviewer (JW) to screen a random selection of $25 \%$ of the titles and abstracts. Full-text articles of potentially eligible articles will be independently assessed by two reviewers (RV and JW). If at any stage discrepancies cannot be resolved through discussion and consensus, a third reviewer (LM) will be consulted.

\section{Data extraction}

A standardised prepiloted data extraction form will be used, with extraction performed independently by two reviewers (RV and JW). Study authors will be contacted if further information is required. Data extracted will include:

1. Study information: author/s, year of baseline data collection and year of publication.

2. Participant characteristics: sample size, study setting, mean age at baseline, relevant eligibility criteria, country/ies of data collection, cohort name (if applicable).

3. Exposure/comparator characteristics: number and spread of measurement occasions, number and nature of drinking consumption/abstention categories.

4. Study design and analysis: approach/es of interest used (ie, study design of interest, combination of traditional study design and analytical method of interest), any additional statistical tests employed, any additional measures taken to enhance causal inference (ie, causal diagrams or tests of robustness), covariates/confounders included in models or otherwise accounted for.
5. Outcomes: health outcome/s assessed and whether these are binary or continuous.

6. Results: findings on the strength and form of the relationship between alcohol and health.

Additional information extracted from MR studies will include: whether there is one study population or pooled data, use of one or two sample MR, instrument used and variance in alcohol consumption explained by the instrument, method of MR analysis used and how it is capable of revealing potential non-linearity and whether there is testing of MR assumptions. Additional information extracted from twin studies will include: whether twins are discordant for exposure or outcome, and whether monozygotic twins are compared with dizygotic twins.

Where both conventional and novel (causal inference method of interest) analyses are conducted, information on both will be extracted. Again, if at any stage discrepancies cannot be resolved through discussion and consensus, a third reviewer (LM) will be consulted.

\section{Risk of bias assessment}

Assessment of bias depends on study design and methodology. As a range of designs and methodologies are expected in the included studies, several existing risk of bias assessment tools will be used. Quality of cohort and case-control studies (including twin cohorts) will be assessed using the relevant Newcastle-Ottawa Scales. ${ }^{26}$ Recently developed risk of bias tools specific to MR studies, natural experiments and family based methods ${ }^{27}$ will be used and adapted where appropriate. One reviewer (RV) will apply the assessment tools to all included studies, with a second reviewer (JW) assessing a random $25 \%$ of the studies to establish scoring accuracy.

\section{Data synthesis}

Descriptive narrative synthesis will be performed. The relationships between alcohol and different health outcomes vary due to mediating causal mechanisms, meaning they are not comparable. As such, in addressing what the included studies find on the relationship between levels of consumption and health, synthesis will be conducted on a per-outcome basis. The heterogeneity of the different analytical approaches means they are not suited to quantitative synthesis. Convergence/lack thereof of findings from each of the various causal inference methods will be assessed in light of the advantages and limitations of each methodology. In evaluating the literature on the effects of moderate alcohol consumption as a whole, the disease burden of each health outcome will be considered.

\section{Assessment of evidence quality}

Popular tools to assess the body of evidence gathered, such as Grading of Recommendations, Assessment, Development and Evaluation $\left(\mathrm{GRADE}^{28}\right)$, are inappropriate here. Given initial 'low quality' ratings are automatically assigned to observational studies, GRADE is of limited use for exposures that do not lend themselves to RCTs ${ }^{29} 30$ (including alcohol consumption ${ }^{31}$ ). Additionally, these 
tools are intended to rate bodies of evidence-such ratings are ill-suited to this review given it is intentionally restricted to a subset of the literature. Consistent with recent reviews focusing on observational studies employing novel causal inference approaches, this review will limit formal assessment of evidence quality to risk of bias. $^{2632}$

\section{Patient and public involvement}

As this is a review of existing published studies, there will be no patient or public involvement in this research.

\section{Ethics and dissemination}

Formal ethics approval is not required given there will be no primary data collection. The results of the study will be disseminated through published manuscripts, conferences and seminar presentations.

Acknowledgements The authors would like to thank Jessica Hughes, librarian at the University of Sydney, for assisting with the literature search.

Contributors RV is the guarantor of the review. RV, MS, TS and LM conceptualised the study and developed the study design and protocol. RV wrote the first draft of the manuscript. RV, MS, TS, JW and LM contributed to, read, revised and approved the final manuscript.

Funding RV is funded by the National Health and Medical Research Council (GNT1190255) and the University of Sydney.

Competing interests None declared.

Patient consent for publication Not required.

Provenance and peer review Not commissioned; externally peer reviewed.

Supplemental material This content has been supplied by the author(s). It has not been vetted by BMJ Publishing Group Limited (BMJ) and may not have been peer-reviewed. Any opinions or recommendations discussed are solely those of the author(s) and are not endorsed by BMJ. BMJ disclaims all liability and responsibility arising from any reliance placed on the content. Where the content includes any translated material, BMJ does not warrant the accuracy and reliability of the translations (including but not limited to local regulations, clinical guidelines, terminology, drug names and drug dosages), and is not responsible for any error and/or omissions arising from translation and adaptation or otherwise.

Open access This is an open access article distributed in accordance with the Creative Commons Attribution Non Commercial (CC BY-NC 4.0) license, which permits others to distribute, remix, adapt, build upon this work non-commercially, and license their derivative works on different terms, provided the original work is properly cited, appropriate credit is given, any changes made indicated, and the use is non-commercial. See: http://creativecommons.org/licenses/by-nc/4.0/.

ORCID iD

Rachel Visontay http://orcid.org/0000-0002-0693-1012

\section{REFERENCES}

1 GBD 2016 Alcohol Collaborators. Alcohol use and burden for 195 countries and territories, 1990-2016: a systematic analysis for the global burden of disease study 2016. Lancet 2018;392:1015-35.

2 Ronksley PE, Brien SE, Turner BJ, et al. Association of alcohol consumption with selected cardiovascular disease outcomes: a systematic review and meta-analysis. BMJ 2011;342:d671.

3 Rehm J, Hasan OSM, Black SE, et al. Alcohol use and dementia: a systematic scoping review. Alzheimers Res Ther 2019;11:1.

4 Kerr WC, Ye Y, Williams E, et al. Lifetime alcohol use patterns and risk of diabetes onset in the National alcohol survey. Alcohol Clin Exp Res 2019;43:262-9.

5 Gémes K, Forsell Y, Janszky I, et al. Moderate alcohol consumption and depression - a longitudinal population-based study in Sweden. Acta Psychiatr Scand 2019;139:526-35.
6 Keyes KM, Calvo E, Ornstein KA, et al. Alcohol consumption in later life and mortality in the United States: results from 9 waves of the health and retirement study. Alcohol Clin Exp Res 2019;43:1734-46.

7 Andreasson S, Chikritzhs T, Dangardt FHH. Evidence about health effect of "moderate" alcohol consumption: Reasons for scepticism and public health implications. In: Alcohol and Society: IOGT-NTO and Swedish Society of MedicineEvidence about health effects of "moderate" alcohol consumption: Reasons for scepticism and public health implilcations. Alcohol Soc 2014;6.

8 Naimi TS, Stockwell T, Zhao J, et al. Selection biases in observational studies affect associations between 'moderate' alcohol consumption and mortality. Addiction 2017;112:207-14.

9 Callinan S, Chikritzhs T, Livingston M. Consistency of drinker status over time: drinking patterns of ex-drinkers who describe themselves as lifetime abstainers. J Stud Alcohol Drugs 2019;80:552-6.

10 Shaper AG, Wannamethee G, Walker M. Alcohol and mortality in British men: explaining the U-shaped curve. Lancet 1988;2:1267-73.

11 Brien SE, Ronksley PE, Turner BJ, et al. Effect of alcohol consumption on biological markers associated with risk of coronary heart disease: systematic review and meta-analysis of interventional studies. BMJ 2011;342:d636.

12 Gepner Y, Golan R, Harman-Boehm I, et al. Effects of initiating moderate alcohol intake on cardiometabolic risk in adults with type 2 diabetes: a 2-year randomized, controlled trial. Ann Intern Med 2015;163:569-79.

13 Field M, Puddephatt J-A, Goodwin L, et al. Benefits of temporary alcohol restriction: a feasibility randomized trial. Pilot Feasibility Stud 2020;6:1-15.

14 Chu L, loannidis JPA, Egilman AC, et al. Vibration of effects in epidemiologic studies of alcohol consumption and breast cancer risk. Int J Epidemiol 2020;49:608-18.

15 Stockwell T, Zhao J, Panwar S, et al. Do "Moderate" Drinkers Have Reduced Mortality Risk? A Systematic Review and Meta-Analysis of Alcohol Consumption and All-Cause Mortality. J Stud Alcohol Drugs 2016;77:185-98.

16 Richmond RC, Al-Amin A, Smith GD, et al. Approaches for drawing causal inferences from epidemiological birth cohorts: a review. Early Hum Dev 2014;90:769-80.

17 Schuler MS, Rose S. Targeted maximum likelihood estimation for causal inference in observational studies. Am J Epidemiol 2017; 185:65-73.

18 Lipsitch M, Tchetgen Tchetgen E, Cohen T. Negative controls: a tool for detecting confounding and bias in observational studies. Epidemiology 2010;21:383.

19 Rehm J, Gmel GE, Gmel G, et al. The relationship between different dimensions of alcohol use and the burden of disease-an update. Addiction 2017;112:968-1001.

20 Watkins TR. Understanding uncertainty and bias to improve causal inference in health intervention research 2019.

21 McQuire C, de Vocht F. Methodological advances to mitigate some of the challenges of research on alcohol and all-cause mortality: commentary on Rehm. Drug Alcohol Rev 2019;38:7-8.

22 Moher D, Shamseer L, Clarke M, et al. Preferred reporting items for systematic review and meta-analysis protocols (PRISMA-P) 2015 statement. Syst Rev 2015;4:1.

23 Moher D, Liberati A, Tetzlaff J, et al. Preferred reporting items for systematic reviews and meta-analyses: the PRISMA statement. PLoS Med 2009;6:e1000097.

24 Clarivate Analytics. Endnote X8, 2016

25 Veritas Health Innovation. Covidence systematic review software. Available: www.covidence.org

26 Wells GA, Shea B, O'Connell D. The Newcastle-Ottawa scale (NOS) for assessing the quality of nonrandomised studies in meta-analyses. Available: http://www.ohri.ca/programs/clinical_epidemiology/oxford. htm

27 Mamluk L, Jones T, ljaz S, et al. Evidence of detrimental effects of prenatal alcohol exposure on offspring birthweight and neurodevelopment from a systematic review of quasi-experimental studies. Int J Epidemiol 2021;49:1972-95.

28 Schünemann H, Brożek J, Guyatt G. Grade Handbook for grading quality of evidence and strength of recommendations. updated October 2013. The grade Working group, 20132013.

29 Arroyave WD, Mehta SS, Guha N, et al. Challenges and recommendations on the conduct of systematic reviews of observational epidemiologic studies in environmental and occupational health. J Expo Sci Environ Epidemiol 2021;31:21-30.

30 World Health Organization. Environmental noise guidelines for the European region World Health organization. regional office for Europe 2018. 
31 NHMRC Clinical Trials Centre at the University of Sydney. Evaluating the evidence on the health effects of alcohol consumption: evidence evaluation report. Sydney NHMRC Clinical Trials Centre; 2019.

32 Mamluk L, Edwards HB, Savović J, et al. Low alcohol consumption and pregnancy and childhood outcomes: time to change guidelines indicating apparently 'safe' levels of alcohol during pregnancy? A systematic review and meta-analyses. BMJ Open 2017;7:e015410.

33 Benedetto U, Head SJ, Angelini GD, et al. Statistical primer: propensity score matching and its alternatives. Eur J Cardiothorac Surg 2018;53:1112-7.

34 Brookhart MA, Wyss R, Layton JB, et al. Propensity score methods for confounding control in nonexperimental research. Circ Cardiovasc Qual Outcomes 2013;6:604-11.

35 Mansournia MA, Etminan M, Danaei G, et al. Handling time varying confounding in observational research. BMJ 2017;359:j4587.

36 Naimi Al, Cole SR, Kennedy EH. An introduction to G methods. Int J Epidemiol 2017;46:756-62.

37 Hernán MA RJ. Causal inference: what if. Boca Raton: Chapman \& Hill/CRC, 2020: 171-82.

38 Williamson T, Ravani P. Marginal structural models in clinical research: when and how to use them? Nephrol Dial Transplant 2017;32:ii84-90.
39 Gunasekara FI, Richardson K, Carter K, et al. Fixed effects analysis of repeated measures data. Int J Epidemiol 2014;43:264-9.

40 Fergusson DM, Boden JM, Horwood LJ. Psychosocial sequelae of cannabis use and implications for policy: findings from the Christchurch health and development study. Soc Psychiatry Psychiatr Epidemiol 2015;50:1317-26.

41 Fergusson DM, Boden JM, Horwood LJ. Tests of causal links between alcohol abuse or dependence and major depression. Arch Gen Psychiatry 2009;66:260-6.

42 Richiardi L, Bellocco R, Zugna D. Mediation analysis in epidemiology: methods, interpretation and bias. Int J Epidemiol 2013;42:1511-9.

43 Pearce N, Lawlor DA. Causal inference-so much more than statistics. Int J Epidemiol 2016;45:1895-903.

44 VanderWeele TJ. Mediation analysis: a practitioner's guide. Annu Rev Public Health 2016;37:17-32.

45 Dunning T. Natural experiments in the social sciences: a designbased approach. Cambridge: Cambridge University Press, 2012.

46 Gage SH, Munafò MR, Davey Smith G, Smith GD. Causal inference in developmental origins of health and disease (DOHaD) research. Annu Rev Psychol 2016;67:567-85.

47 Davies NM, Holmes MV, Davey Smith G. Reading Mendelian randomisation studies: a guide, glossary, and checklist for clinicians. BMJ 2018;362:k601. 